\title{
PLANAR POLYNOMIAL FOLIATIONS
}

\author{
STEPHEN SCHECTER AND MICHAEL F. SINGER
}

\begin{abstract}
Let $P(x, y)$ and $Q(x, y)$ be two real polynomials of degree $<n$ with no common real zeros. The solution curves of the vector field $\dot{x}=P(x, y), \dot{y}=$ $Q(x, y)$ give a foliation of the plane. The leaf space $\mathcal{L}$ of this foliation may not be a hausdorff space: there may be leaves $L, L^{\prime} \in \mathcal{L}$ which cannot be separated by open sets. We show that the number of such leaves is at most $2 n$ and construct an example, for each even $n>4$, of a planar polynomial foliation of degree $n$ whose leaf space contains $2 n-4$ such leaves.
\end{abstract}

1. Introduction. In [2], L. Markus considered the following problem: Let $P(x, y), Q(x, y)$ be polynomials of degree < $n$. If we assume $P(x, y), Q(x, y)$ have no common real zeros, then the solution curves of the vector field

$$
\dot{x}=P(x, y), \quad \dot{y}=Q(x, y)
$$

give a foliation of the plane, which we shall call a planar polynomial foliation of degree $n$. Call two foliations of $\mathbf{R}^{2}$ equivalent if there is a homeomorphism of $\mathbf{R}^{2}$ that carries the curves of one foliation to those of the other. The problem is to get a bound, depending on $n$, on the number of equivalence classes. In his paper, Markus obtained such a bound. A key step in his calculation is to bound the number of inseparable leaves of the foliation. A leaf $L$ is said to be inseparable if there exists another leaf $L^{\prime}$ such that any two neighborhoods of $L$ and $L^{\prime}$ in the leaf space intersect; see Figure 1. Markus showed that there are at most $6 n$ inseparable

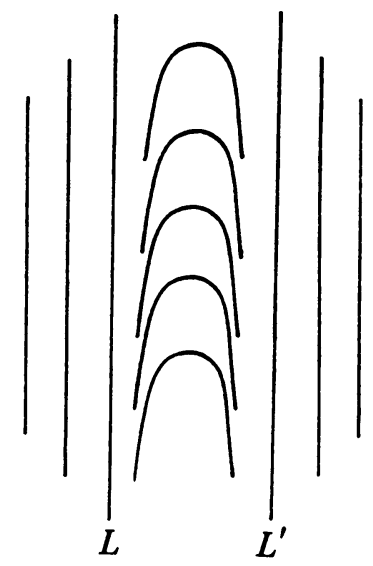

FIgURE 1. $L$ and $L^{\prime}$ are inseparable leaves

Received by the editors June 21, 1979.

AMS (MOS) subject classifications (1970). Primary 57D30; Secondary 58F99.

Key words and phrases. Planar polynomial vector field, foliation, inseparable leaves. 
leaves. In $\$ 2$, we show that the number of inseparable leaves is actually at most $2 n$. In $\S 3$, we show how to construct, for any even $n>4$, a planar polynomial foliation of degree $n$ with $2 n-4$ inseparable leaves. We do not know if there are planar polynomial foliations of degree $n, n \geqslant 4$, with more than $2 n-4$ inseparable leaves.

2. Maximum number of inseparable leaves. Given any polynomial vector field on $\mathbf{R}^{n}$, one can construct a "singular point at infinity" such that analysis of this singular point tells us the behavior of the polynomial vector field at infinity. In $\mathbf{R}^{2}$ the construction is as follows:

Let $x^{2}+y^{2}+z^{2}=1$ be the unit sphere in $\mathbf{R}^{3}$. Identify the $x y$-plane with the tangent plane $z=-1$. Let $\pi$ be stereographic projection from the north pole to the plane $z=-1$, and let $\tilde{\pi}$ be stereographic projection from the south pole to the plane $z=1$. We have a map $\tilde{\pi} \circ \pi^{-1}$ from the plane $z=-1$ minus $(0,0,-1)$ to the plane $z=1$ minus $(0,0,1)$ given by

$$
u=4 x /\left(x^{2}+y^{2}\right), \quad v=4 y /\left(x^{2}+y^{2}\right) .
$$

Here we have used $u$ and $v$ as coordinates on the plane $z=1$.

Applying (2.1) to (1.1), we get a vector field on the $u v$-plane minus $(0,0)$ of the form

$$
\dot{u}=\tilde{P}(u, v) /\left(u^{2}+v^{2}\right)^{n}, \quad \dot{v}=\tilde{Q}(u, v) /\left(u^{2}+v^{2}\right)^{n}
$$

where $\tilde{P}$ and $\tilde{Q}$ are polynomials whose lowest degree terms are of degree $>n+2$. Then the vector field

$$
\dot{u}=\tilde{P}(u, v), \quad \dot{v}=\tilde{Q}(u, v)
$$

defined on the entire $u v$-plane has a singularity at $(0,0)$ and has the same solution curves as (2.2) on the $u v$-plane minus $(0,0)$. Thus the flow of $(2.3)$ in a deleted neighborhood of $(0,0)$ is conjugate to the flow of $(1.1)$ in a neighborhood of infinity.

Now we specialize to the case where (1.1) has no zeros.

Proposition 2.1. Inseparable leaves of the foliation defined by (1.1) correspond to boundaries of hyperbolic sectors at $(0,0)$ of $(2.3)$.

Proof. The proposition is almost an immediate consequence of the well-known description of singular points of analytic vector fields in the plane found, for example, in [1]. We recall that a singular point of an analytic vector field in the plane is either (a) a center, (b) a spiral node, or (c) composed of a finite number of elliptic, hyperbolic, and parabolic sectors. Let $c$ be any solution curve of (1.1). Then $c$ approaches infinity at both ends (otherwise there would be a zero of (1.1) in the finite plane). It follows that the singular point at infinity cannot be of the form (a) or (b). If neither end of $c$ corresponds to the boundary of a hyperbolic sector of the singular point at infinity, then both ends of $c$ correspond to curves that approach the singular point at infinity in parabolic or elliptic sectors. It follows easily that $c$ can be separated from any other leaf in the leaf space of (1.1). 
TheOREM 2.2. Let $P(x, y)$ and $Q(x, y)$ be real polynomials of degree $<n$ with no common real zeros. Then the foliation of $\mathbf{R}^{2}$ defined by (1.1) has at most $2 n$ inseparable leaves.

Proof. The singular point $(0,0)$ of $(2.3)$ must be of index 2 , since we could easily construct a vector field on the sphere having this point as its only singularity. A well-known formula of Bendixson [1, p. 511] says that if $e$ is the number of elliptic sectors, $h$ the number of hyperbolic sectors, and $i$ the index of a singular point, then $e-h+2=2 i$. In our case,

$$
e-h=2 .
$$

Consider a big circle $\Gamma: x^{2}+y^{2}=R^{2}$ in the $x y$-plane. $\tilde{\pi} \circ \pi^{-1}(\Gamma)$ is a small circle $\gamma$ in the $u v$-plane that traverses each hyperbolic and elliptic sector at $(0,0)$ of (2.3). Each such sector contains a point of $\gamma$ where the vector field (2.3) is tangent to $\gamma$. These points correspond to points $(x, y)$ in the $x y$-plane where $x^{2}+y^{2}=R^{2}$ and $x P+y Q=0$. By Bezout's Theorem there are at most $2 n+2$ such points, so

$$
e+h<2 n+2 \text {. }
$$

Combining (2.4) and (2.5) we have $h<n$. Each hyperbolic sector has two boundary curves, so by Proposition 2.1 , there are at most $2 n$ inseparable leaves of the foliation defined by (1.1).

3. An example. Let $n=2 m \geqslant 4$. We will describe a polynomial foliation of the plane of degree $n$ with $2 n-4$ inseparable leaves.

Let

$$
\dot{x}=P(x, y)=\prod_{k=1}^{m} A_{k}(x, y), \quad \dot{y}=Q(x, y)=\prod_{k=1}^{m} B_{k}(x, y)
$$

where

$$
\begin{aligned}
& A_{1}(x, y)=x(x+2 m y)+1, \\
& A_{k}(x, y)=(k x+y)(x+k y)+a_{k}, \quad k=2, \ldots, m, \\
& B_{1}(x, y)=y(x+2 m y)+1, \\
& B_{k}(x, y)=[k(x+1)+(y-1)][(x+1)+k(y-1)]+b_{k}, \quad k=2, \ldots, m .
\end{aligned}
$$

The $a_{k}$ and $b_{k}$ are positive constants to be chosen so that $P^{-1}(0) \cap Q^{-1}(0)=\varnothing$. Notice $P$ and $Q$ are polynomials of degree $n$.

First we explain geometrically how to choose $a_{k}$ and $b_{k}$ recursively. Notice $P^{-1}(0)=A_{1}^{-1}(0) \cup \ldots \cup A_{m}^{-1}(0), Q^{-1}(0)=B_{1}^{-1}(0) \cup \ldots \cup B_{m}^{-1}(0)$, so both $P^{-1}(0)$ and $Q^{-1}(0)$ are unions of hyperbolas. $A_{1}^{-1}(0)$ is a hyperbola in the second and fourth quadrants asymptotic to $x=0$ and $y=-x / 2 m . B_{1}^{-1}(0)$ is a hyperbola in the second and fourth quadrants asymptotic to $y=0$ and $y=-x / 2 m$. $A_{1}^{-1}(0)$ $\cap B_{1}^{-1}(0)=\varnothing$. The remaining $A_{k}^{-1}(0)$ and $B_{k}^{-1}(0)$ are also to be hyperbolas in the second and fourth quadrants; this forces $a_{k}$ and $b_{k}$ to be positive. Notice each $A_{k}^{-1}(0)$ is to be asymptotic to $y=-k x$ and $y=-x / k$, with center at $(0,0)$; each $B_{k}^{-1}(0)$ is to be asymptotic to $y-1=-k(x+1)$ and $y-1=-(x+1) / k$, with center at $(-1,1)$. Therefore, the vertices of each hyperbola $A_{k}^{-1}(0)$ and $B_{k}^{-1}(0)$ will 
be on the line $y=-x$. To choose $a_{2}$ and $b_{2}$, notice that for any $a_{2}>0, A_{2}^{-1}(0) \cap$ $B_{1}^{-1}(0)=\varnothing$, and for $b_{2}$ big enough, $A_{1}^{-1}(0) \cap B_{2}^{-1}(0)=\varnothing$. Choose $a_{2}$ and $b_{2}$ this $\mathrm{big}$, and so that the vertices of $A_{2}^{-1}(0)$ and $B_{2}^{-1}(0)$ are ordered as follows, from left to right: $B_{2}^{-1}(0), A_{2}^{-1}(0), B_{2}^{-1}(0), A_{2}^{-1}(0)$. Then we have $\left(A_{1}^{-1}(0) \cup A_{2}^{-1}(0)\right) \cap$ $\left(B_{1}^{-1}(0) \cup B_{2}^{-1}(0)\right)=\varnothing$. See Figure 2 .

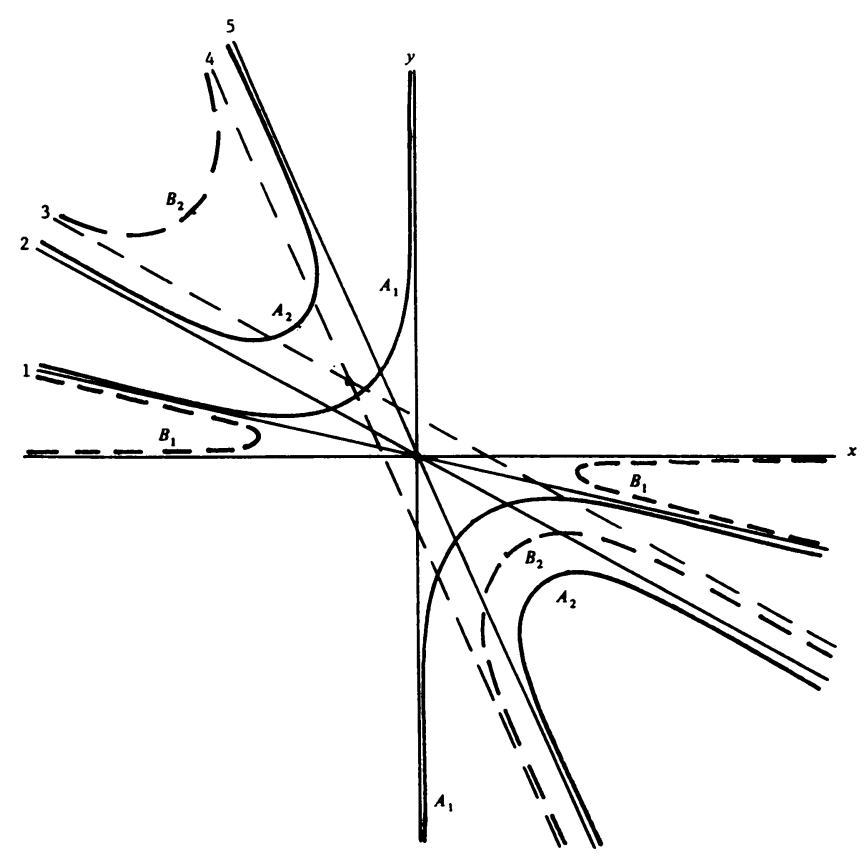

Figure 2

$$
\begin{aligned}
& \text { 1. } y=-\frac{1}{2 m} x \quad \text { 2. } y=-\frac{1}{2} x \quad \text { 3. } y+1=-\frac{1}{2}(x-1) \\
& \text { 4. } y+1=-2(x-1) \text { 5. } y=-2 x
\end{aligned}
$$

Branches of $A_{i}^{-1}(0)$ and $B_{i}^{-1}(0)$ are indicated by $A_{i}, B_{i}$

Proceeding recursively, assume $a_{2}, \ldots, a_{k-1}$ and $b_{2}, \ldots, b_{k-1}$ have been chosen so that $\cup_{i=1}^{k-1} A_{i}^{-1}(0) \cap \cup_{i=1}^{k-1} B_{i}^{-1}(0)=\varnothing$. For $a_{k}$ and $b_{k}$ big enough, $A_{k}^{-1}(0) \cap$ $\cup_{i=1}^{k-1} B_{i}^{-1}(0)=\varnothing$ and $\cup_{i=1}^{k-1} A_{i}^{-1}(0) \cap B_{k}^{-1}(0)=\varnothing$. Choose $a_{k}$ and $b_{k}$ this big and so that the vertices of $A_{k}^{-1}(0)$ and $B_{k}^{-1}(0)$ are ordered as follows, from left to right: $B_{k}^{-1}(0), A_{k}^{-1}(0), B_{k}^{-1}(0), A_{k}^{-1}(0)$. Then we have $\cup_{i=1}^{k} A_{i}^{-1}(0) \cap \cup_{i=1}^{k} B_{i}^{-1}(0)=\varnothing$.

We will determine the number of inseparable leaves of our foliation by analyzing the behavior of (3.1) at infinity. Rather than construct a singularity at infinity, we will pass from (3.1) to a circle at infinity. The method we use goes back to Poincaré.

Suppose we have a polynomial vector field

$$
\begin{aligned}
& \dot{x}=P(x, y)=P_{n}(x, y)+P_{n-1}(x, y)+\cdots+P_{0}(x, y), \\
& \dot{y}=Q(x, y)=Q_{n}(x, y)+Q_{n-1}(x, y)+\cdots+Q_{0}(x, y),
\end{aligned}
$$


where each $P_{i}$ and $Q_{i}$ is homogeneous of degree $i$ and $P_{n}^{2}+Q_{n}^{2} \neq 0$. Let $S$ denote the unit circle parametrized by the angle $\theta,-\pi / 2<\theta<3 \pi / 2$. Consider the map $S \times \mathbf{R} \rightarrow \mathbf{R}^{2}$ given by

$$
x=r^{-1} \cos \theta, \quad y=r^{-1} \sin \theta .
$$

Of course, this map is not defined on $S \times\{0\} . S \times(0, \infty)$ (also $S \times(-\infty, 0)$ ) is mapped diffeomorphically onto $R^{2}-\{0\} . S \times\{0\}$ is the "circle at infinity".

Pulling back (3.2) by (3.3) we get

$$
\begin{aligned}
& \dot{r}=-\sum_{i=0}^{n} r^{2-i}\left(\cos \theta P_{i}(\cos \theta, \sin \theta)+\sin \theta Q_{i}(\cos \theta, \sin \theta)\right), \\
& \dot{\theta}=\sum_{i=0}^{n} r^{1-i}\left(\cos \theta Q_{i}(\cos \theta, \sin \theta)-\sin \theta P_{i}(\cos \theta, \sin \theta)\right) .
\end{aligned}
$$

Now for the vector field (3.1) we have $\cos \theta Q_{n}(\cos \theta, \sin \theta)-\sin \theta P_{n}(\cos \theta, \sin \theta)$ $\equiv 0$. Therefore, if we pull back (3.1) by (3.3) and multiply by $r^{n-2}$, we get

$$
\begin{aligned}
& \dot{r}=-\sum_{i=0}^{n} r^{n-i}\left(\cos \theta P_{i}(\cos \theta, \sin \theta)+\sin \theta Q_{i}(\cos \theta, \sin \theta)\right), \\
& \dot{\theta}=\sum_{i=0}^{n-1} r^{n-i-1}\left(\cos \theta Q_{i}(\cos \theta, \sin \theta)-\sin \theta P_{i}(\cos \theta, \sin \theta)\right) .
\end{aligned}
$$

Notice that (3.5) defines an analytic vector field on all $S \times \mathbf{R}$. The solution curves of (3.5) in $S \times(\mathbf{R}-\{0\})$ are the same as those of (3.4), up to reparametrization. Moreover, since $n$ is even, we also have that the solution curves of (3.5) are parametrized in the same sense as those of (3.4). The behavior of (3.5) near $S \times\{0\}$ corresponds to the behavior of (3.1) at infinity.

From (3.5) we have that

$$
\dot{r} \mid S \times\{0\}=-\left[\cos \theta P_{n}(\cos \theta, \sin \theta)+\sin \theta Q_{n}(\cos \theta, \sin \theta)\right] .
$$

Therefore, the vector field (3.5) is transverse to $S \times\{0\}$ except at the roots of (3.6). These roots correspond to linear factors of the homogeneous polynomial

$$
x P_{n}(x, y)+y Q_{n}(x, y)=\left(x^{2}+y^{2}\right)(x+2 m y) \prod_{k=2}^{m}(k x+y)(x+k y) .
$$

Therefore, the roots of equation (3.6) with $-\pi / 2<\theta<\pi / 2$ are

$$
\begin{aligned}
& \theta=\operatorname{Tan}^{-1}(-1 / 2 m) ; \\
& \theta=\operatorname{Tan}^{-1}(-1 / 2), \operatorname{Tan}^{-1}(-1 / 3), \ldots, \operatorname{Tan}^{-1}(-1 / m) ; \\
& \theta=\operatorname{Tan}^{-1}(-2), \operatorname{Tan}^{-1}(-3), \ldots, \operatorname{Tan}^{-1}(-m) .
\end{aligned}
$$

The roots of (3.6) with $\pi / 2<\theta<3 \pi / 2$ are constructed by adding $\pi$ to each of these. All factors of (3.7) have multiplicity one, so $r \mid S \times\{0\}$ changes sign at each root.

To study $\dot{\theta} \mid S \times\{0\}$, we first note from (3.5) that

$$
\dot{\theta} \mid S \times\{0\}=\cos \theta Q_{n-1}(\cos \theta, \sin \theta)-\sin \theta P_{n-1}(\cos \theta, \sin \theta) .
$$

The roots of (3.8) correspond to linear factors of the homogeneous polynomial $x Q_{n-1}(x, y)-y P_{n-1}(x, y)$. We compute from (3.1) that $P_{n-1}(x, y) \equiv 0$, 


$$
\begin{aligned}
& Q_{n-1}(x, y) \\
& \quad=y(x+2 m y) \sum_{k=2}^{m}[(k x+y)(1-k)+(k-1)(x+k y)] \prod_{j \neq k}(j x+y)(x+j y) .
\end{aligned}
$$

Therefore,

$$
\begin{aligned}
& x Q_{n-1}(x, y)-y P_{n-1}(x, y) \\
& \quad=x y(x+2 m y) \sum_{k=2}^{m}[(k x+y)(1-k)+(k-1)(x+k y)] \prod_{j \neq k}(j x+y)(x+j y) .
\end{aligned}
$$

We will now find all the factors of (3.9). (1) Three factors are $x, y$, and $x+2 m y$. (2) Another is $y-x$, since

$$
(k x+y)(1-k)+(k-1)(x+k y)=(1-k)^{2}(y-x) .
$$

(3) Notice that when $x=1,(3.9)$ becomes

$$
y(1+2 m y) \sum_{k=2}^{m}[(k+y)(1-k)+(k-1)(1+k y)] \prod_{j \neq k}(j+y)(1+j y) .
$$

We can compute that (3.10) is nonzero at $y=-2,-3, \ldots,-m$ and at $y=-1 / 2$, $-1 / 3, \ldots,-1 / m$; the sign of (3.10) at $y=-2$ and at $y=-1 / 2$ is the same (namely, $(-1)^{m+1}$ ); for each $k=2, \ldots, m-1$, the sign of (3.10) at $y=-k$ is opposite that at $y=-(k+1)$; and for each $k=2, \ldots, m-1$, the sign of (3.10) at $y=-1 / k$ is opposite that at $y=-1 /(k+1)$. There must be $2 m-4$ roots of (3.10) between $y=-m$ and $y=-1 / m$ to account for these sign changes. Thus we have $2 m-4$ additional factors of (3.9). Since (3.9) has degree $2 m$, we have found all its factors.

We conclude that $\dot{\theta} \mid S \times\{0\}$ changes sign at $\theta=-\pi / 2,0, \pi / 2, \pi$, $\operatorname{Tan}^{-1}(-1 / 2 m), \pi+\operatorname{Tan}^{-1}(-1 / 2 m) ;$ at $\theta=\pi / 4,5 \pi / 4$; and, for each $k=$ $1,2, \ldots, m-1$, between $\theta=\operatorname{Tan}^{-1}(-k)$ and $\theta=\operatorname{Tan}^{-1}(-(k+1))$, between $\theta=$ $\pi+\operatorname{Tan}^{-1}(-k)$ and $\theta=\pi+\operatorname{Tan}^{-1}(-(k+1))$, between $\theta=\operatorname{Tan}^{-1}(-1 / k)$ and $\theta=\operatorname{Tan}^{-1}(-1 /(k+1))$, and between $\theta=\pi+\operatorname{Tan}^{-1}(-1 / k)$ and $\theta=\pi+$ $\operatorname{Tan}^{-1}(-1 /(k+1))$.

We will identify the inseparable leaves of the foliation defined by (3.1) by looking at the vector field (3.5) on $S \times[0, \infty)$. At the points of $S \times\{0\}$ where $\dot{r}=0$ we have the following germs. At $\theta=\operatorname{Tan}^{-1}(-1 / 2 m)$ and at $\theta=\pi+$ $\operatorname{Tan}^{-1}(-1 / 2 m)$ there are nodes. At $\theta=\operatorname{Tan}^{-1}(-2), \operatorname{Tan}^{-1}(-3), \ldots, \operatorname{Tan}^{-1}(-m)$ the solution curve of $(3.5)$ that passes through $(\theta, 0)$ opens toward the positive $r$-direction. See Figure 3. Each of these curves through $(\theta, 0)$ corresponds to two inseparable leaves of (3.1). At $\theta=\pi+\operatorname{Tan}^{-1}(-2), \pi+\operatorname{Tan}^{-1}(-3), \ldots, \pi+$ $\operatorname{Tan}^{-1}(-m)$, and at $\theta=\operatorname{Tan}^{-1}(-1 / 2), \operatorname{Tan}^{-1}(-1 / 3), \ldots, \operatorname{Tan}^{-1}(-1 / 2 m)$, the solution curve of $(3.5)$ that passes through $(\theta, 0)$ opens toward the negative $r$-direction. At $\theta=\pi+\operatorname{Tan}^{-1}(-1 / 2), \pi+\operatorname{Tan}^{-1}(-1 / 3), \ldots, \pi+\operatorname{Tan}^{-1}(-1 / m)$ the solution 


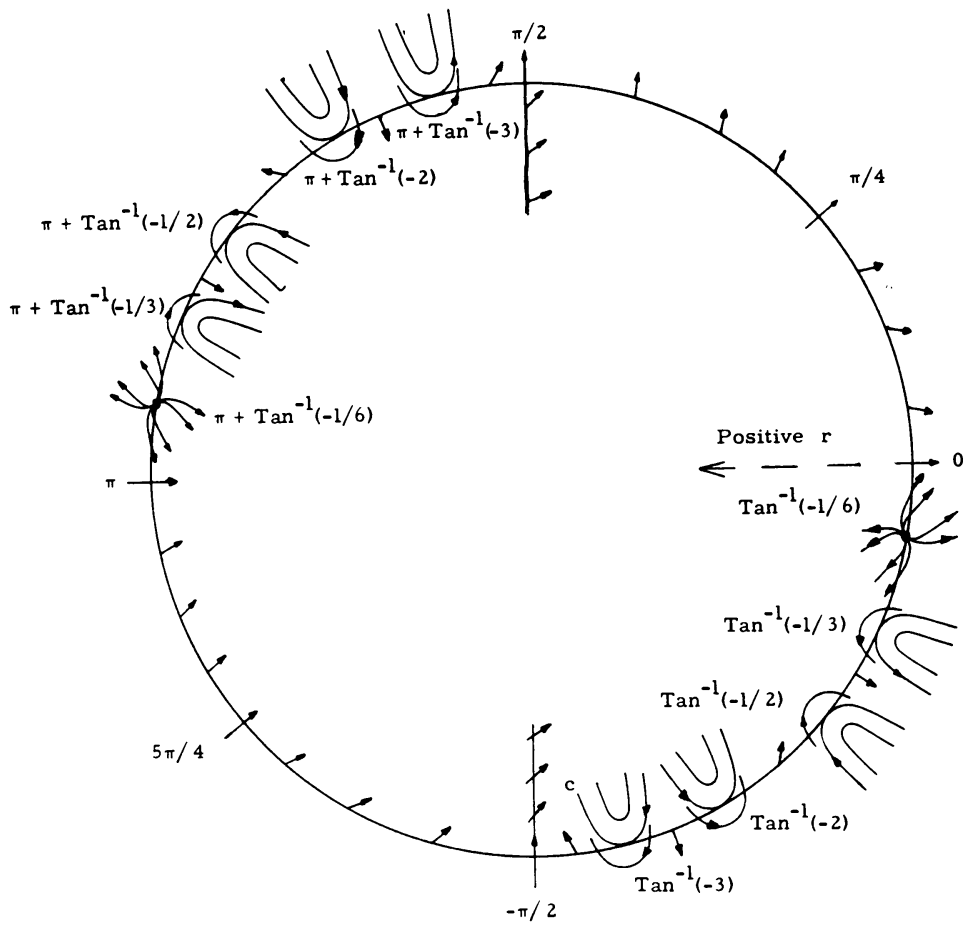

FIgURE 3

The vector field (3.5) near $S \times\{0\}$ with $m=3$

curve of $(3.5)$ that passes through $(\theta, 0)$ again opens toward the positive $r$-direction. These curves again each correspond to two inseparable leaves of (3.1). Thus we have identified $2 n-4$ inseparable leaves of (3.1), provided they are all distinct.

We conclude with a geometric argument that shows these inseparable leaves to be distinct. Let $\varphi: J \rightarrow S \times \mathbf{R}, \varphi(t)=(\theta(t), r(t))$, be the solution curve of (3.5) through $\left(\operatorname{Tan}^{-1}(-m), 0\right)$ with $\varphi(0)=\left(\operatorname{Tan}^{-1}(-m), 0\right)$. Here $J$ is the largest interval on which $\varphi$ can be defined. Let $a$ be the greatest number $<\infty$ such that $\varphi(-a, 0)$ $\subset S \times(0, \infty)$, and let $b$ be the greatest number $<\infty$ such that $\varphi(0, b) \subset S \times$ $(0, \infty)$. Let $c=\varphi(0, b) ; c$ is the "lower half" of the solution curve of (3.5) through $\left(\operatorname{Tan}^{-1}(-m), 0\right)$ (see Figure 3$)$. Let $c^{\prime}$ be the corresponding curve in the $x y$-plane. Then after choosing an initial point on $c^{\prime}, c^{\prime}$ has a natural parametrization $(x(t), y(t))$. As $t \rightarrow-\infty, x(t) \rightarrow \infty$ and $y(t) / x(t) \rightarrow-m$. From (3.1), $\dot{x}(0, y)>0$ for all $y \in \mathbf{R}$, so $c^{\prime}$ lies entirely in the half-plane $x>0$. Since (3.1) has no zeros in the finite plane, $c^{\prime}$ approaches infinity at both ends. It follows that $c$ lies in $(-\pi / 2, \pi / 2)$ $\times(0, \infty)$ and approaches $S \times\{0\}$ at both ends.

Any solution curves of (3.5) that approaches $S \times\{0\}$ must approach a single point of $S \times\{0\}$. We can compute that $\dot{r}(\theta, 0)>0$ for $-\pi / 2<\theta<\operatorname{Tan}^{-1}(-m)$. Therefore, $\lim _{t \rightarrow b} \theta(t) \geqslant \operatorname{Tan}^{-1}(-m)$. If $\lim _{t \rightarrow b} \theta(t)=L, \operatorname{Tan}^{-1}(-m)<L<$ $\operatorname{Tan}^{-1}(-1 / 2 m)$, then consider the closed curve $\Gamma=\varphi(0, b) \cup\left[\operatorname{Tan}^{-1}(-m), L\right] \times$ $\{0\}$. We see from Figure 3 that the vector field (3.5) is nonzero on $\Gamma$ and that its 
index around $\Gamma$ is $>1$. Therefore, (3.5) has a zero inside $\Gamma$, which is impossible. It follows that $\lim _{t \rightarrow b} \theta(t)>\operatorname{Tan}^{-1}(-1 / 2 m)$. We conclude that $c$ divides its complement in $S \times(0, \infty)$ into two components, one of which contains $\varphi(-a, 0)$ and the solution curves of (3.5) through $\left(\operatorname{Tan}^{-1}(-2), 0\right),\left(\operatorname{Tan}^{-1}(-3), 0\right), \ldots$, $\left(\operatorname{Tan}^{-1}(-m+1), 0\right)$, while the other contains the solution curves of (3.5) through $\left(\pi+\operatorname{Tan}^{-1}(-1 / 2), 0\right),\left(\pi+\operatorname{Tan}^{-1}(-1 / 3), 0\right), \ldots,\left(\pi+\operatorname{Tan}^{-1}(-1 / m), 0\right)$. Thus we can consider these two sets of curves separately. Now we can again use an index argument to show that no two of the inseparable leaves we have found can be identical.

\section{REFERENCES}

1. A. A. Andronov et al., Qualitative theory of second order dymamic systems, Wiley, New York, 1973.

2. L. Markus, Topological types of polynomial differential equations, Trans. Amer. Math. Soc. 171 (1972), 157-178.

Departaient of Mathematics, North Carolna State Universtty, Rnueigh, North Carolina 27607 (Current address of S. Schecter and M. F. Singer)

School of Mathematics, The Institute for Advanced Study, Princeton, New Jersey 08540 